\title{
Clinical Expression of Calcium Sensing Receptor Polymorphism (A986S) in Normocalcemic and Asymptomatic Hyperparathyroidism
}

Authors

Affiliations

\section{G. Díaz-Soto' , E. Romero', J. L. P. Castrillón², O. I. Jauregui', D. de Luis Román}

Endocrinology and Nutrition Service, University Hospital of Valladolid, Centro de Investigación de Endocrinología y Nutrición-University of Valladolid, Valladolid, Spain

Internal Medicine Service, Centro de Investigación de Endocrinología y Nutrición, Hospital Universitario Rio Hortega, Valladolid, Spain
Key words

- parathyroid hormone

- calcium sensing receptor

- calcium

- hyperparathyroidism

received 10.04.2015 accepted 27.07.2015

\section{Bibliography}

DOI http://dx.doi.org/

10.1055/s-0035-1559723

Published online:

September 2, 2015

Horm Metab Res 2016;

48: 163-168

(c) Georg Thieme Verlag KG

Stuttgart · New York

ISSN 0018-5043

\section{Correspondence}

\section{Dr. G. Díaz-Soto}

Hospital Clinico Universitario de Valladolid

Servicio de Endocrinología

Edificio Rondilla

Calle Rondilla de Santa Teresa

s/n. 47005

Valladolid

Spain

Tel.: + 34/983/420000

Ext.: 21615

Fax: +34/983/42 0000

diazsotogonzalo@gmail.com

\section{Abstract}

$\nabla$

Normocalcemic and asymptomatic hyperparathyroidism diagnosis are becoming more common. However, their pathophysiology is incompletely known. The aim of the present study was to evaluate the clinical effect of calcium-sensing receptor polymorphism (A986S) in normocalcemic and asymtomatic HPT. Prospective study conducted with 61 consecutive normocalcemic and asymptomatic HPT patients was followed up during a minimum period of 1 year. Secondary causes of hyperparathyroidism were ruled out. Calcium and phosphorus metabolism parameters were evaluated in at least 2 determinations during follow-up to classify as normocalcemic or asymptomatic hyperparathyroidism. Bone mineral density and A986S polymorphism genotype were also analyzed. Thiry-eight patients $(62.3 \%)$ had the genotype A986A, and $23(36.7 \%)$ patients had A986S (20 patients, $32.8 \%$ ) or S986S (3 patients, $4.9 \%$ ). Age, sex, and genotype distribu-

\section{Introduction}

\section{$\nabla$}

Traditionally, diagnosis of primary hyperparathyroidism (HPT) is based on the demonstration of high total and/or ionized serum calcium associated with an increased or unsuppressed serum parathyroid hormone (PTH) concentration [1]. However, in the last years, the understanding of HPT has evolved, and it is now characterized as having several distinctive clinical forms [2]. Routine measurement of calcium levels and generalization of PTH determination has led to the identification of a new clinical entity named normocalcemic primary hyperparathyroidism (NPHPT). NPHPT is characterized by persistently normal total and ionized serum calcium concentrations and consistently elevated PTH levels, after ruling out secondary causes of PTH eleva- tions were comparable in both normocalcemic and asymptomatic hyperparathyroidism. In normocalcemic patients, $\mathrm{S}$ allele genotype was associated to statistically significant higher level of intact PTH: 92.0 (SD 18.5) vs. 110.6 (SD 24.4) $\mathrm{pg} / \mathrm{ml}, \mathrm{p}<0.05$; and remained significant after adjustment by multiple linear regression. In asymptomatic hyperparathyroidism, A986A genotype resulted in a statistically significant higher level of intact PTH, alkaline phosphatase and procollagen amino-terminal propeptide; but only serum calcium remained as an independent predictor of serum intact PTH levels after a multiple linear regression. Bone mineral densitometry between genotypes did not show statistically significant differences. A986S polymorphism of CaSR is an independent predictor of PTH level in normocalcemic hyperparathyroidism patients, but not in asymptomatic hyperparathyroidism. More studies are needed to evaluate the effect of other polymorphisms in normocalcemic and asymptomatic hyperparathyroidism.

tion, such as renal disease, vitamin D deficiency or familial hypercalcemia hypocalciuria [3]. NPHPT has generated a considerable scientific interest since its first formal recognition in the Third International Workshop on the Management of Asymptomatic Primary Hyperparathyroidism in 2008 [4]. However, this entity remains incompletely described, particularly regarding its pathophysiology, epidemiology, natural history, and management; although its diagnosis is becoming more common in different clinical settings.

NPHPT has been proposed as an early or a mild variety of classical HPT. However, contradictory publications exist for NPHPT evolution to a hypercalcemic HPT [5-7], or against this progression in general population [8]. Furthermore, interpretation of the data is biased by the differing definition of NPHPT and the methods used to 
exclude secondary hyperparathyroidism. This reflects the lack of evidence about natural history and pathophysiology of NPHPT.

Previous studies suggest that NPHPT may be related to target tissue resistance to PTH and modulated by postmenopausal estrogen deficiency [9]. However, this hypothesis has not been further studied. Single nucleotide polymorphism (SNP) in Calcium Sensing Receptor (CaSR) - rs17251221 (A986S) - has been linked to PTH resistance and higher calcium levels in adult women $[10,11]$, but its effect has never been studied in mild HPT patients. A986S is localized in exon 7 of the CaSR gene, coding the intracellular domain of the receptor [12], and it is associated to a relative loss of CaSR function [13].

The aim of the present work was to address the effect of A986S polymorphism on calcium and phosphorus metabolism regulation and its complications in patients with normocalcemic or asymptomatic HPT.

\section{Patients and Methods \\ $\nabla$ \\ Patients and procedure}

We conducted a prospective study that enrolled 61 consecutive NPHPT and asymptomatic HPT patients. NPHPT and asymptomatic HPT patients were recruited consecutively from the clinical facilities of the Calcium Metabolism Unit (Endocrinology and Nutrition Service-IEN) at the University Hospital of Valladolid, Spain in 2013. To assure the correct classification of HPT, patients were followed up during a minimum period of 1 year, during which intact plasma PTH, 25 hydroxyvitamin D (25vitD), and serum total calcium, albumin-corrected total calcium, and ionized calcium were analyzed in at least 2 determinations.

NPHPT was defined as persistently normal total, albumin corrected and ionized serum calcium concentrations and consistently elevated PTH levels [14]. Asymptomatic HPT patients were defined as those with consistently elevated or unsuppressed PTH and at least one determination of total, albumin corrected or ionized serum calcium over the upper limit of reference [14]. Secondary causes of PTH elevation were strictly ruled out, such as renal disease (glomerular filtration rate $<60 \mathrm{ml} / \mathrm{min}$ ), vitamin D deficiency $(25 \mathrm{VitD}<30 \mathrm{ng} / \mathrm{ml})$, genetic disorders as familial hypercalcemic hypocalciuria, significant hypercalciuria (urinary calcium $>250 \mathrm{mg} / 24 \mathrm{~h}$ in females and $>300 / 24 \mathrm{~h}$ in males), other metabolic bone diseases and thiazide diuretic, lithium or bisphosphonates treatment. Nephrolithiasis and fractures were documented by history and review of medical records. Secondary elevation of PTH and classical and symptomatic HPT patients were excluded from the study.

The study was carried out according to the guidelines laid down in the Declaration of Helsinki and all procedures were approved by the local Ethical Committee. Written informed consent was obtained from all patients. A standardized questionnaire was used to collect the clinical and laboratory data. Clinical features were recorded after patients visited to the hospital. Privacy and confidentiality was ensured for collected data.

\section{Biochemical assays and genotyping of (CaSR) gene polymorphism}

Biochemical assays were performed in fasting condition at the end of the follow-up period. It included: intact PTH (reference range, $10-65 \mathrm{pg} / \mathrm{ml}$ ), total serum calcium (reference range, $8.5-$ $10.5 \mathrm{mg} / \mathrm{dl}$ ), ionized calcium (reference range, $1.00-1.38 \mathrm{mmol} / \mathrm{l}$ ) and albumin corrected calcium using the formula: corrected calcium $=(4 \text {-serum albumin })^{*} 0.8$-serum calcium, phosphorus, magnesium, 25vitD (reference range 3-70 ng/ml), and 1,25 dihydroxyvitamin $\mathrm{D}$ (1,25vitD). All plasma and urine determination were analyzed in a Cobas Hitachi Platform (Roche Diagnostics $\mathrm{GmbH}$, Mannheim, Germany), except ionized calcium that was analyzed by a 9180 Electrolyte Analyzer Platform (Roche Diagnostics GmbH, Mannheim, Germany) using a calcium ion-selective electrode in anaerobic sample collection and handling conditions. Bone Turnover Markers: osteocalcin, procollagen type 1 amino-terminal propeptide (P1NP) and $\beta$-crosslaps, as well as 24-h urine calcium and phosphate levels were analyzed. Bone mineral density (BMD) was assessed by dual-energy X-ray absorptiometry (Lunar, General Electrics Healthcare, USA) at the lumbar spine (L1-L4), proximal femur and femoral neck, for absolute BMD, T-scores, and Z-score.

DNA extraction was realized with a lysate of blood cells (Geneall; Biorad, Seoul, Korea). Buffy coats of nucleated cells obtained from anticoagulated blood (ACD or EDTA) were resuspended in $15 \mathrm{ml}$ polypropylene centrifugation tubes with $3 \mathrm{ml}$ of nuclei lysis buffer. The cell lysates were digested overnight at $37^{\circ} \mathrm{C}$ with $0.2 \mathrm{~mL}$ of $10 \%$ sodium dodecyl sulfate and $0.5 \mathrm{ml}$ of a protease $\mathrm{K}$ solution. After digestion was complete, $1 \mathrm{ml}$ of saturated $\mathrm{NaCl}$ (approximately 6M) was added to each tube and shaken vigorously for $15 \mathrm{~s}$, followed by centrifugation at 2500 revolutions/ min for $15 \mathrm{~min}$. The precipitated protein pellet was left at the bottom of the tube, and the supernatant containing the DNA was transferred to another $15 \mathrm{ml}$ polypropylene tube. The precipitated DNA strands were removed with a plastic spatula or pipette and transferred to a $1.5 \mathrm{ml}$ microcentrifuge tube containing 100-200 pl TE buffer. The DNA was allowed to dissolve $2 \mathrm{~h}$ at $37^{\circ} \mathrm{C}$ before quantitating.

Oligonucleotide primers were designed with the Sequenom Assay Design v4 (SEQUENOM, Inc., San Diego, CA, USA). The polymerase chain reaction (PCR) was carried out with $20 \mathrm{ng}$ of genomic DNA, $0.1 \mu \mathrm{L}$ of each oligonucleotide primer (primer forward: 5'-ACGTTGGATGGTGACTCCTCTCCTGTATTT-3' and reverse 5'-ACGTTGGATGAGATGGCACTGCTTCTTAAC-3' in a $2 \mu \mathrm{l}$ final volume (Termociclador Lifetecnologies, LA, CA, USA). DNA was denaturated at $85^{\circ} \mathrm{C}$ for $5 \mathrm{~min}$; this was followed by 45 cycles of denaturation at $95^{\circ} \mathrm{C}$ for $15 \mathrm{~s}$, and annealing at $58.1^{\circ} \mathrm{C}$ for $45 \mathrm{~s}$. The PCR were run in a $2 \mu$ final volume containing $0.1 \mu$ of iPLEx Termination mix (Bio-Rad ${ }^{\circledR}$, San Diego, CA, USA) with hot start Taq DNA polymerase. Hardy-Weinberg equilibrium was assessed with a statistical test (Chi-square) to compare our expected and observed counts. The 2 variants were in Hardy-Weinberg equilibrium.

\section{Statistical analysis}

Sample size was calculated to detect differences over $15 \mathrm{pg} / \mathrm{ml}$ in PTH level with $90 \%$ power and $5 \%$ significance $(n=40)$. The results are expressed as mean and standard deviation (SD). The distribution of variables was analyzed with KolmogorovSmirnov test. Quantitative variables with normal distribution were analyzed with a 2-tailed Student's $t$-test. Nonparametric variables were analyzed with the Friedman and Wilcoxon tests. A Chi square test was used to evaluate the Hardy-Weinberg equilibrium. Clinically relevant variables were included into stepwise multivariate linear regression analysis to detect the independent variables that were significantly related to PTH. All covariates significantly associated with PTH or those which 


\begin{tabular}{|c|c|c|c|}
\hline & NPHPT & Asymptomatic HPT & $\mathbf{p}$ \\
\hline Age (years) & $63(S D 11)$ & $66(S D 10)$ & ns \\
\hline Sex (female:male) & $34: 7$ & $16: 4$ & ns \\
\hline Intact PTH (pg/ml) & 103 (SD 25) & $152($ SD 65) & 0.001 \\
\hline 25-Hydroxy Vit D (ng/ml) & 34.0 (SD 10.9) & $22.9($ SD 17.0) & 0.005 \\
\hline 1,25-Dihydroxy Vit D (pg/ml) & 47.2 (SD 15.8) & 35.6 (SD 15.8) & ns \\
\hline Total serum calcium (mg/dl) & 9.56 (SD 0.39) & $10.6(S D 0.52)$ & $<0.001$ \\
\hline Albumin corrected calcium (mg/dl) & 9.23 (SD 0.43) & 10.23 (SD 0.49) & $<0.001$ \\
\hline Ionized calcium (mmol/l) & 1.22 (SD 0.04) & $1.36($ SD 0.06$)$ & $<0.001$ \\
\hline Serum phosphate (mg/dl) & 3.39 (SD 0.48) & 2.9 (SD 0.60) & 0.015 \\
\hline Serum magnesium $(\mathrm{mg} / \mathrm{dl})$ & $2.12(S D 0.15)$ & $1.91($ SD 0.20$)$ & 0.002 \\
\hline Alkaline phosphatase (UI/I) & 65.4 (SD 16.3) & 79.9 (SD 21.9) & 0.021 \\
\hline Osteocalcin (ng/ml) & $19.2($ SD 5.2) & 32.5 (SD 13.2) & 0.008 \\
\hline Procollagen type 1 amino-terminal ( $\mathrm{ng} / \mathrm{ml})$ & 36.1 (SD 14.6) & 56.0 (SD 26.0) & 0.048 \\
\hline B crosslaps $(\mathrm{ng} / \mathrm{ml})$ & $0.29($ SD 0.15$)$ & 0.54 (SD 0.35) & 0.032 \\
\hline 24-H urine calcium (mg/24h) & $218.4($ SD 120.6) & 251.8 (SD 177.7) & ns \\
\hline 24-H urine phosphate $(\mathrm{mg} / 24 \mathrm{~h})$ & 817.1 (SD 318.7) & 785.6 (SD 289.2) & ns \\
\hline Creatinine clearance $(\mathrm{ml} / \mathrm{min})$ & $89.3(\mathrm{SD} 28.1)$ & $84.3($ SD 22.1) & ns \\
\hline Phosphate tubular reabsorption & 80.9 (SD 6.37) & 73.0 (SD 11.1) & 0.038 \\
\hline $\begin{array}{l}\text { Maximum rate of tubular phosphate } \\
\text { reabsorption }\end{array}$ & 2.80 (SD 0.55) & 2.01 (SD 0.43) & 0.025 \\
\hline
\end{tabular}

Table 1 Comparison baseline features between normocalcemic hyperparathyroidism (NPHPT) and asymptomatic hyperparathyroidism. improved model fit were retained in the final model. A p-value of less than 0.05 was considered statistically significant. The statistical analysis was performed for the combined S986S and A986S as a group and genotype A986A as second group, with a dominant model. All analysis were carried out using the Statistical Package for Social Sciences (SPSS) version 20.0 (SPSS, Chicago, IL, USA)

\section{Results}

A total of 61 HPT patients ( $82 \%$ females) with a mean age of 65 years old $(S D=11)$ were enrolled in the study. 41 patients $(83 \%$ female, mean age 63 years old, $S D=13$ ) were classified as NPHPT, 20 patients were classified as asymtomatic HPT ( $80 \%$ female, mean age 66 years old $S D=10$ ) after a minimum period of 1 year of follow-up ( $\bullet$ Table 1 ). All women included were postmenopausal.

Asymptomatic HPT showed statistically significant higher levels of intact PTH, total and albumin corrected serum calcium, ionized calcium, alkaline phosphatase and bone turnover markers (osteocalcin, P1NP and B crosslaps) and significant lower levels of 25VitD, serum phosphate and magnesium, phosphate tubular reabsorption and maximum rate of tubular phosphate reabsorption than NPHPT group ( $\odot$ Table 1). However, no differences between groups were demonstrated in bone mineral density in any site or score analyzed ( $\odot$ Table 2 ).

SNP rs17251221 analysis from CaSR gene showed 38 HPT patients (62.3\%) with A986A genotype (wild type), and 23 (36.7\%) patients with A986S (20 patients, 32.8\%) or S986S (3 patients, $4.9 \%$ ) (S allele carriers). Age, sex and genotype distributions were comparable in both NHPT and asymptomatic HPT ( $\odot$ Table 3 ).

- Table 3 shows differences in metabolic variables depending on SNP genotype (A986A vs. A986S or S986S) in the NPHPT and the asymptomatic hyperparathyroidism groups.

In patients with NPHPT, S allele carriers were only associated to a statistically significant higher level of intact PTH. Although albumin corrected serum calcium and ionized calcium showed higher levels in A986S or S986S genotype, they did not reach statistical significance.
Table 2 Comparison bone mineral densitometry between normocalcemic hyperparathyroidism (NPHPT) and asymptomatic hyperparathyroidism.

\begin{tabular}{|c|c|c|c|}
\hline & NPHPT & $\begin{array}{l}\text { Asymptomatic } \\
\text { HPT }\end{array}$ & \\
\hline Femoral neck $\left(\mathrm{g} / \mathrm{cm}^{2}\right)$ & 0.766 (SD 0.081) & $0.813(S D 0.081)$ & ns \\
\hline Proximal femur $\left(\mathrm{g} / \mathrm{cm}^{2}\right)$ & 0.845 (SD 0.127) & $0.855(S D 0.100)$ & ns \\
\hline L1-L4 Lumbar spine $\left(\mathrm{g} / \mathrm{cm}^{2}\right)$ & 0.994 (SD 0.185) & 0.996 (SD 0.177) & ns \\
\hline Femoral neck T-Score & $-1.810($ SD 0.640$)$ & $-1.500($ SD 0.744$)$ & ns \\
\hline Proximal femur T-Score & -1.359 (SD 0.943) & -1.306 (SD 0.851) & ns \\
\hline L1-L4 Lumbar spine T-Score & -1.526 (SD 1.476) & -1.606 (SD 1.426) & ns \\
\hline Femoral neck Z-Score & -0.342 (SD 0.508) & -0.380 (SD 0.741) & ns \\
\hline Proximal femur Z-Score & -0.254 (SD 0.765) & $-0.473($ SD 0.723$)$ & ns \\
\hline L1-L4 Lumbar spine Z-Score & -0.139 (SD 1.508) & -0.393 (SD 1.496) & ns \\
\hline
\end{tabular}

In those patients classified as asymptomatic hyperparathyroidism, A986A genotype resulted in a statistically significant higher level of intact PTH, alkaline phosphatase and P1NP. Furthermore, A986A allele was associated to nonsignificant higher levels of 1,25 VitD, osteocalcin, B crosslaps and total and albumin corrected serum and ionized calcium.

In NPHPT group, the associations analyzed by multiple linear regression between the intact PTH and the A986S or S986S genotype remained significant after adjustment for albumin, serum calcium, glomerular filtration rate, serum phosphate and 25vitD. ( 0 Table 4) However, in asymptomatic HPT group only serum calcium remained as an independent predictor of serum intact PTH levels ( $\bullet$ Table 5).

Bone mineral densitometry did not show statistically significant differences (data not shown) between among A986A and A986S or S986S genotypes in NPHPT and asymptomatic hyperparathyroidism groups

\section{Discussion}

In this study, we have explored the effect of A986S polymorphism of the CaSR in the clinical features of NPHPT and asymptomatic HPT. 
Table 3 Comparison in metabolic features between A986A and A986S or S986S genotype in normocalcemic hyperparathyroidism (NPHPT) and asymptomatic hyperparathyroidism groups.

\begin{tabular}{|c|c|c|c|c|c|c|}
\hline & & NPHPT & & & iptomatic HPT & \\
\hline & A986A & A986S & $\mathbf{p}$ & A986A & A986S & $\mathbf{p}$ \\
\hline & & S986S & & & S986S & \\
\hline Number patients & 24 & 17 & ns & 14 & 6 & ns \\
\hline Age (years) & 66.8 (SD 12.2) & 66.6 (SD 9.4) & ns & 68.2 (DS 9.1) & 73.4 (DS 12.6) & 0.025 \\
\hline Intact PTH (pg/ml) & 92.0 (SD 18.5) & 110.6(SD 24.4) & 0.024 & 170.2 (SD 71.4) & $104.4(30.9)$ & 0.015 \\
\hline 25-Hydroxy Vit D (ng/ml) & 33.4 (SD 12.8) & 34.0 (SD 8.4) & ns & $25.0($ SD 20.8) & 20.6 (SD 9,6) & ns \\
\hline 1,25-Dihydroxy Vit D (pg/ml) & 46.6 (SD 15.3) & 41.4 (SD 15.6) & ns & 42,7 (SD 17,2) & 26.7 (SD 8.1) & 0.070 \\
\hline Total serum calcium (mg/dl) & 9.55 (SD 0.42) & 9.54 (SD 0.41) & ns & 10.60 (SD 0.38) & 10.40 (SD 0.66) & ns \\
\hline Albumin corrected calcium (mg/dl) & 9.20 (SD 0.49) & 9.35 (SD 0.22) & ns & 10.21 (SD 0.43) & 10.03 (SD 0.52) & ns \\
\hline Ionized calcium (mmol/l) & 1.21 (SD 0.05) & $1.22(0.045)$ & ns & 1.37 (SD 0.07) & $1.34(0.03)$ & ns \\
\hline Serum phosphate (mg/dl) & 3.36 (SD 0.46) & 3.41 (SD 0.54) & ns & 3.00 (SD 0.68) & 2.89 (SD 0.44) & ns \\
\hline Serum magnesium (mg/dl) & 2.12 (SD 0.17) & 2.10 (SD 0.14) & ns & 1.93 (SD 0.16) & 1.90 (SD 0.29) & ns \\
\hline Alkaline phosphatase (UI/I) & 65.2 (SD 20.7) & 67.6 (SD 12.9) & ns & 85.8 (SD 23.2) & 65.0 (SD 16.4) & 0.045 \\
\hline Osteocalcin (ng/ml) & 18.3 (SD 5.09) & 20.7 (SD 5.57) & ns & 35.6 (SD 13.8) & 18.0 (SD 5.6) & 0.090 \\
\hline Procollagen type 1 amino-terminal (ng/ml) & 33.7 (SD 13.6) & 39.7 (SD 16.3) & ns & 65.7 (SD 24.6) & 22.7 (SD 12.2) & 0.049 \\
\hline B crosslaps $(\mathrm{ng} / \mathrm{ml})$ & $0.28(S D 0.15)$ & $0.30($ SD 0.16$)$ & ns & 0.62 (SD 0.37) & $0.16(S D 0.15)$ & 0.090 \\
\hline 24-H urine calcium (mg/24h) & 231.4 (SD 140.9) & 212.0 (SD 111.9) & ns & 262.2 (SD 190.4) & 204.4 (SD 175.0) & ns \\
\hline 24-H urine phosphate (mg/24h) & 761.9 (SD 296.7) & 834.7 (SD 368.8) & ns & 782.0 (SD 262.9) & 727.7 (SD 162.1) & ns \\
\hline Creatinine clearance (ml/min) & 84.8 (SD 24.3) & 85.8 (SD 21.9) & ns & $81.4($ SD 22.0) & 95.3 (SD 37.3) & ns \\
\hline Phosphate tubular reabsorption & 79.8 (SD 7.57) & 83.1 (SD 4.61) & ns & 75.5 (SD 10.21) & 74.3 (SD 10.58) & ns \\
\hline $\begin{array}{l}\text { Maximum rate of tubular phosphate } \\
\text { reabsorption }\end{array}$ & 2.75 (SD 0.57) & $2.76(0.66)$ & ns & $2.18($ SD 0.43$)$ & 1.93 (SD 0.058) & ns \\
\hline
\end{tabular}

ns: Not significant

Table 4 Multivariate regression analysis of A986S effect on intact PTH in NPHPT.

\begin{tabular}{lcrrrc} 
& Coefficient (B) & \multicolumn{1}{c}{ SE } & R & t & P \\
Serum Albumin & -21.011 & 18.822 & -0.189 & -1.116 & 0.276 \\
Serum Phosphate & -13.766 & 8.087 & -0.294 & -1.702 & 0.102 \\
\hline 25VitD & 0.292 & 0.361 & 0.137 & 0.809 & 0.427 \\
Glomerular & -0.194 & 0.211 & -0.169 & -0.918 & 0.368 \\
filtration rate & & & & & \\
Serum calcium & 2.287 & 10.494 & 0.041 & 0.218 & 0.829 \\
A986S & -20.663 & 7.716 & -0.455 & -2.678 & 0.013
\end{tabular}

Shown in the table are the estimate for the regression coefficient (B) with standard error (SE), standardized variant (R), t statistic, and probability (p) by stepwise analyses with a constant $B$ of $235 \pm 144$

NPHPT has received a considerable scientific interest in last years, in fact, conclusions of the Fourth International Workshop on the Management of Asymptomatic Primary Hyperparathyroidism have been recently published [14]. This guideline recommends a careful diagnose of NPHPT with normal total and ionized serum calcium levels associated to high intact PTH values confirmed on at least 2 further occasions over a period of 3-6 months, after ruling out any known etiology for a secondary elevation of PTH [15]. Although it is accepted that some normocalcemic patients could become hypercalcemic in the future, most diagnosed patients appear to be stable over time with persistently elevated PTH levels and normal serum calcium concentrations. However, NPHPT has been classically considered as a frustrated form or an early stage of the classic HPT [16], although its clinical evolution to hypercalcemic forms is not completely confirmed [8].

In our study, all patients were carefully evaluated in fasting conditions with intact PTH and total and albumin corrected serum calcium, as well as with ionized calcium, after excluding secondary HPT causes. All patients were followed up during a mini-
Table 5 Multivariate regression analysis of A986S effect on intact PTH in asymptomatic hyperparathyroidism.

\begin{tabular}{lrrrrc} 
& Coefficient (B) & \multicolumn{1}{c}{ SE } & R & t & P \\
Serum Albumin & -129.138 & 105.293 & -0.421 & -1.226 & 0.251 \\
Serum Phosphate & -1.957 & 22.605 & -0.022 & -0.087 & 0.933 \\
25VitD & 0.063 & 0.800 & 0.021 & 0.079 & 0.939 \\
Glomerular & -0.411 & 0.534 & -0.233 & -0.769 & 0.461 \\
filtration rate & & & & & \\
Serum calcium & 71.614 & 31.309 & 0.610 & 2.287 & 0.048 \\
A9865 & 39.147 & 25.856 & 0.342 & 1.514 & 0.164
\end{tabular}

Shown in the table are the estimate for the regression coefficient (B) with standard error (SE), standardized variant (R), t statistic, and probability $(\mathrm{p})$ by stepwise analyses with a constant $B$ of $-26.5 \pm 117$

mum period of 1 year to confirm their correct diagnosis of NPHPT or asymptomatic HPT. What is more, NPHPT was defined in the most strictly way to avoid misclassifications in NPHPT group. Thus, this study may reflect the natural history of both NPHPT and asymptomatic HPT.

The comparison between NPHPT and asymptomatic HPT reflected that asymptomatic HPT shows most evolved HPT clinical features ( $\bullet$ Table 1). Asymptomatic HPT showed higher levels of intact PTH, calcium and bone turnover markers; and lower levels of serum phosphate, magnesium, phosphate tubular reabsorption, and maximum rate of tubular phosphate reabsorption. These characteristics are similar to those found in classical hypercalcemic HPT; thus, asymptomatic HPT could be considered as a mild form of classical HPT [5-7]. However, the most evolved nature of asymtomatic HPT was not reflected in bone mineral density ( $\odot$ Table 2 ), maybe due to the nonlineal and complex multifactorial determinants on bone mineral density (PTH and vitamin D levels, calcium intake, physical exercise, etc.) or to a mild deleterious effect secondary to NPHPT $[17,18]$. On the other hand, BMD was not evaluated at distal third of the 
radius that could be shown differences between groups, as a predominant cortical bone. However, last studies have discussed this classical deleterious effect in NPHPT and asymptomatic HPT in contrast to classical HPT [18].

Previous studies have suggested that NPHPT may be related to target tissue resistance to the actions of PTH and modulated by postmenopausal estrogen deficiency [9]. Although it is a highly interesting hypothesis, especially in those patients with no evolution to hypercalcemic forms, it has not been fully clarified. The CaSR is a G-protein-coupled cell-surface receptor that modulates extracellular calcium concentration by regulation of PTH secretion in the parathyroid glands [19]. In the present study we have evaluated A986S polymorphism of the CaSR as a determinant of PTH resistance in NPHPT and asymptomatic HPT.

A986S polymorphism has been extensively linked to PTH resistance and higher calcium levels in population based studies $[10,11,20]$. However, only few studies exist that evaluate the effect of A986S polymorphism in HPT, and it has never been studied neither in mild forms of HPT nor in NPHPT.

The prevalence and nonstatistically significant distribution between groups of S allele carriers found in our study (36.7\%) were similar to those previously published in general population and HPT patients [21]. Thus, it supports that A986S CaSR variant do not seem to be a major genetic determinants for the development of primary HPT, even in earlier or asymptomatic forms [22]. However, the present study supports the pathogenic effect of A986S polymorphism, especially in NPHPT.

In NPHPT, only A986S and S986S genotypes were independent predictors of PTH level even after adjusting for major confounding factors as vitamin D level and serum calcium concentrations. Therefore, PTH levels in NPHPT may be partially regulated by A986S polymorphism, acting as a resistance factor due to a relative loss of CaSR function, as previously described [13]. Although no differences in serum and urine metabolic and BMD parameters between $A$ and $S$ allele carriers were found, this could be explained by the small number of patients to find minimum differences between groups.

On the other hand, in asymptomatic HPT, serum calcium was the only independent predictors of PTH level after adjusting for major confounding factors, including A986S genotype. Thus, A986S genotype did not affect HPT features as previously described in classical HPT [22,23]. However, in bivariate analysis those patients with A986A genotype showed clinical features consistent with a more evolved HPT: significantly higher levels of intact PTH and bone turnover markers, with nonsignificant higher levels of serum calcium. This striking difference could be explained by the design of the present study: all classical forms of HPT were excluded, and NPHPT was defined in the most restricted way to avoid misclassification in the normocalcemic group, in contrast to previously published literature. Besides, these differences could be justified not only by variations in the techniques of determination of calcium levels, but also due to the natural history of a dynamic disease such as HPT [24]. Furthermore, previous studies assessing different SNPs in classic HPT have shown opposite effects for each specific polymorphism evaluated [25].

In the present study, the number of S986S homozygotes was too small to exclude the possibility of a gene dosage effect, however there are some published articles that biologically support this hypothesis $[10,11,20]$. Genome-wide association studies are required to confirm the effect of $A 986 S$ and other gene polymor- phisms on NPHPT and asymptomatic HPT. Finally, as our patients were recruited in a Calcium Metabolism Unit, this could lead to a referral bias, as observed in virtually all populations that have been described with NPHPT [3].

In conclusion, A986S polymorphism of CaSR is an independent predictor of PTH level in NPHPT patients, probably acting as a resistance factor due to a relative loss of CaSR function in the $\mathrm{PTH}$ regulation. However, this effect was not observed in asymptomatic HPT. Further studies are needed to evaluate the role of SNP in CASR and other serum calcium regulation genes in the natural history and the evolution of NPHPT to hypercalcemic forms.

\section{Fundings}

$\nabla$

This research did not receive any specific grant from any funding agency in the public, commercial or not-for-profit sector.

\section{Acknowledgements}

$\nabla$

We are indebted to Dr. Isabel Martinez Pino for statistical support.

\section{Conflict of Interest}

$\nabla$

The authors declare no conflict of interest.

\section{References}

1 Díaz-Soto G, Julián MT, Puig-Domingo M. Normocalcemic primary hyperparathyroidism: a newly emerging disease needing therapeutic intervention. Hormones (Athens) 2012; 11: 390-396

2 Bilezikian JP, Khan AA, Potts JT. Guidelines for the management of asymptomatic primary hyperparathyroidism: summary statement from the third international workshop. J Clin Endocrinol Metab 2009; 94: 335-339

3 Cusano NE, Silverberg SJ, Bilezikian JP. Normocalcemic primary hyperparathyroidism. J Clin Densitom Off J Int Soc Clin Densitom 2013; 16: 33-39

4 Eastell R, Arnold A, Brandi ML, Brown EM, D'Amour P, Hanley DA, Rao DS, Rubin MR, Goltzman D, Silverberg SJ, Marx SJ, Peacock M, Mosekilde $L$, Bouillon R, Lewiecki EM. Diagnosis of asymptomatic primary hyperparathyroidism: proceedings of the third international workshop. J Clin Endocrinol Metab 2009; 94: 340-350

5 Silverberg SJ, Bilezikian JP. "Incipient" primary hyperparathyroidism: a "forme fruste" of an old disease. J Clin Endocrinol Metab 2003; 88: 5348-5352

6 Tordjman KM, Greenman Y, Osher E, Shenkerman G, Stern N. Characterization of normocalcemic primary hyperparathyroidism. Am J Med 2004; 117: 861-863

7 Lowe H, McMahon DJ, Rubin MR, Bilezikian JP, Silverberg SJ. Normocalcemic primary hyperparathyroidism: further characterization of a new clinical phenotype. J Clin Endocrinol Metab 2007; 92: 3001-3005

8 García-Martín A, Reyes-García R, Muñoz-Torres M. Normocalcemic primary hyperparathyroidism: one-year follow-up in one hundred postmenopausal women. Endocrine 2012; 42: 764-766

9 Maruani G, Hertig A, Paillard M, Houillier P. Normocalcemic primary hyperparathyroidism: evidence for a generalized target-tissue resistance to parathyroid hormone. J Clin Endocrinol Metab 2003; 88: 4641-4648

10 Cole DE, Peltekova VD, Rubin LA, Hawker GA, Vieth R, Liew CC, Hwang DM, Evrovski J, Hendy GN. A986S polymorphism of the calcium-sensing receptor and circulating calcium concentrations. Lancet 1999; 353: $112-115$

11 Cole DE, Vieth R, Trang HM, Wong BY, Hendy GN, Rubin LA. Association between total serum calcium and the A986S polymorphism of the calcium-sensing receptor gene. Mol Genet Metab 2001; 72: 168-174 
12 Heath $H$, Odelberg S, Jackson CE, Teh BT, Hayward N, Larsson C, Buist NR, Krapcho KJ, Hung BC, Capuano IV, Garrett JE, Leppert MF. Clustered inactivating mutations and benign polymorphisms of the calcium receptor gene in familial benign hypocalciuric hypercalcemia suggest receptor functional domains. J Clin Endocrinol Metab 1996; 81: $1312-1317$

13 O'Seaghdha CM, Yang Q Glazer NL, Leak TS, Dehghan A, Smith AV, Kao WHL, Lohman K, Hwang S-J, Johnson AD, Hofman A, Uitterlinden $A G$, Chen Y-DI, Brown EM, Siscovick DS, Harris TB, Psaty BM, Coresh J, Gudnason V, Witteman JC, Liu YM, Kestenbaum BR, Fox CS, Köttgen A. Common variants in the calcium-sensing receptor gene are associated with total serum calcium levels. Hum Mol Genet 2010; 19: 4296-4303

14 Bilezikian JP, Brandi ML, Eastell R, Silverberg SJ, Udelsman R, Marcocci C, Potts JT. Guidelines for the Management of Asymptomatic Primary Hyperparathyroidism: Summary Statement from the Fourth International Workshop. J Clin Endocrinol Metab 2014; 99: 3561-3569

15 Eastell R, Brandi ML, Costa AG, D'Amour P, Shoback DM, Thakker RV. Diagnosis of Asymptomatic Primary Hyperparathyroidism: Proceedings of the Fourth International Workshop. J Clin Endocrinol Metab 2014; 99: 3570-3579

16 Bilezikian JP, Silverberg SJ. Normocalcemic primary hyperparathyroidism. Arq Bras Endocrinol Metabol 2010; 54: 106-109

17 Hernández JL, Olmos JM, Pariente E, Nan D, Martínez J, Llorca J, Valero C, Obregón E, González-Macías J. Influence of vitamin D status on vertebral fractures, bone mineral density, and bone turnover markers in normocalcemic postmenopausal women with high parathyroid hormone levels. J Clin Endocrinol Metab 2013; 98: 1711-1717

18 Koumakis E, Souberbielle J-C, Sarfati E, Meunier M, Maury E, Gallimard $E$, Borderie D, Kahan A, Cormier C. Bone Mineral Density Evolution After Successful Parathyroidectomy in Patients With Normocalcemic Primary Hyperparathyroidism. J Clin Endocrinol Metab 2013; 98: 3213-3220
19 Brown EM, Hebert SC. Calcium-receptor-regulated parathyroid and renal function. Bone 1997; 20: 303-309

20 He Y, Han L, Li W, Shu X, Zhao C, He Y, Bi M, Li Y, Sun C. Effects of the calcium-sensing receptor A986S polymorphism on serum calcium and parathyroid hormone levels in healthy individuals: a meta-analysis. Gene 2012; 491: 110-115

21 Miedlich S, Lamesch P, Mueller A, Paschke R. Frequency of the calciumsensing receptor variant $A 986 \mathrm{~S}$ in patients with primary hyperparathyroidism. Eur J Endocrinol 2001; 145: 421-427

22 Cetani F, Borsari S, Vignali E, Pardi E, Picone A, Cianferotti L, Rossi G, Miccoli P, Pinchera A, Marcocci C. Calcium-sensing receptor gene polymorphisms in primary hyperparathyroidism. J Endocrinol Invest 25: 614-619

23 Corbetta S, Eller-Vainicher C, Filopanti M, Saeli P, Vezzoli G, Arcidiacono T, Loli P, Syren ML, Soldati L, Beck-Peccoz P, Spada A. R990G polymorphism of the calcium-sensing receptor and renal calcium excretion in patients with primary hyperparathyroidism. Eur J Endocrinol 2006; 155: 687-692

24 Nordenström E, Katzman P, Bergenfelz A. Biochemical diagnosis of primary hyperparathyroidism: Analysis of the sensitivity of total and ionized calcium in combination with PTH. Clin Biochem 2011; 44: 849-852

25 Kanzawa M, Sugimoto T, Kobayashi T, Kobayashi A, Chihara K. Parathyroid hormone gene polymorphisms in primary hyperparathyroidism. Clin Endocrinol (Oxf) 1999; 50: 583-588 